\title{
P45. Oleanolic Acid Delivery using Biodegradable Nanoparticles for Cancer Therapy
}

Man Kwun Wai DeDe; Dr. Philip Kwok; Dr. Jenny Lam Department of Pharmacology and Pharmacy, The University of Hong Kong

Oleanolic acid $(O A)$ is a naturally occurring pentacyclic triterpenoid widely found in Chinese herbs. It has been extensively studied owing to the beneficial effects such as hepatoprotection, anti-inflammatory and it is recently found to have anti-tumor ability. However, resembling the other hydrophobic drugs, it has poor water solubility and therefore very limited intestinal absorption when administrated orally. Investigations concerning the delivery of OA have been carried out to enhance its dissolution and bioavailability. Nanoparticulate systems involve the reduction of drug particle size into nano-scale thereby increasing the interfacial surface area. As a result, absorption of drug in the body system could be enhanced. Biodegradable polymer-based system can be metabolized by the body system into harmless compounds so it is regarded safe and promising. This study was conducted to evaluate the efficacy of four types of biodegradable copolymers as potential OA delivery nanoparticulate system.

Co-polymers consisting a hydrophilic block: poly(ethylene) glycol (PEG) and a hydrophobic block of either poly(lactic-co-glycolic) acid (PLGA) or poly(lactic acid) (PLA) were used to fabricate a novel nanoformulation to improve $O A$ bioavailability. The $O A$-loaded mPEG-(D,L)PLA, mPEG-(L)PLA, mPEG-(D,L)PLGA and mPEG(L)PLGA nanoparticles (NPs), prepared by nanoprecipitation, were observed to be spherical in shape under transmission electron microscope. Results indicated that narrow size distributed NPs with mean hydrodynamic diameter of $200 \pm 16.0,233 \pm 13.9,211 \pm 10.1$ and $229 \pm 10.1 \mathrm{~nm}$ respectively were obtained. mPEG-(D,L)PLA NPs attained the highest encapsulation of $O A$ with $75.8 \%$ efficiency while mPEG-(L)PLGA NPs displayed the lowest encapsulation efficiency at $40.8 \%$. The in vitro anti-tumoral activity was evaluated on A549, lung carcinoma epithelial cell lines using MTT assay. All the four types of OA-loaded NPs demonstrated better anti-cancer ability than saturated OA in medium. Moreover, NPs without OA exhibited significantly lower cytotoxicity towards A549 indicating induced cell death was due to the effective delivery of $O A$ as an anti-cancer therapeutic agent.

Email: u3001488@hku.hk, pclkwok@hku.hk, jkwlam@hku.hk 